NASA Technical Memorandum 84346

NASA-TM-84346 19830015075

\title{
Ablation of Carbonaceous Materials in a Hydrogen-Helium Arc-Jet Flow
}

C. Park, J. H. Lundell, M. J. Green, W. Winovich and M. A. Covington

March 1983

\section{LIBRARY COPY \\ APR ? R 1983 \\ LANGLEY RESEARCH CENTER \\ LIBRARY, NASA \\ HAMPTON, VIRGINIA}

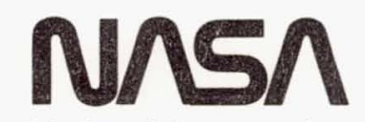

National Aeronautics and

Space Administration 


\title{
Ablation of Carbonaceous Materials in a Hydrogen-Helium Arc-Jet Flow
}

\author{
C. Park \\ J. H. Lundell \\ M. J. Green \\ W. Winovich \\ M. A. Covington, Ames Research Center, Moffett Field, California
}

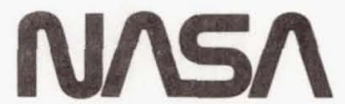

National Aeronautics and

Space Administration 


\section{$\underline{\text { Abstract }}$}

The stagnation-point ablation rates of a graphite, a carbon-carbon composite, and four carbonphenolic materials are measured in an arc-jet wind tunnel with a $50 \%$ hydrogen- $50 \%$ helium mixture as the test gas. Flow environments are determined through measurements of static and impact pressures, heat-transfer rates to a calorimeter, and radiation spectra, and through numerical calculation of the flow through the wind tunnel, spectra, and heattransfer rates. The environments so determined are: impact pressure $\cong 3 \mathrm{~atm}$, Mach number $\cong 2.1$, convective heat-transfer rate $\cong 14 \mathrm{~kW} / \mathrm{cm}^{2}$, and radiative heat-transfer rate $\cong 7 \mathrm{~kW} / \mathrm{cm}^{2}$ in the absence of ablation. Ablation rates are determined from the measured rates of mass loss and recession of the ablation specimens. Compared with the predicted ablation rates obtained by running RASLE and CMA codes, the measured rates are higher by about $15 \%$ for all tested materials.

\section{Introduction}

Later in this decade, a spacecraft, named Galileo Probe, is expected to enter the atmosphere of the planet Jupiter. Its heat shield is made of two types of carbon-phenolic materials, i.e., chopmolded carbon-phenolic (CMCP) for the spherical nose and tape-wrapped carbon-phenolic (TWCP) for the conical frustum. Thickness of the heat shield is determined so as to accommodate the theoretically predicted entry heating environments. ${ }^{1}$ Two groups of computer codes are involved in this prediction, i.e., flow-environment codes such as HYVIS, COLTS, and RASLE, and the materials-response code CMA. ${ }^{1,2}$ The three flow-environment codes use nearly the same physical models and predict approximately the same heat-transfer rates to the heat shield surface. ${ }^{2}$

In addition to the numerical calculations, the performance of the heat-shield materials is determined experimentally. Experiments are needed because of the uncertainties in the calculations: the theoretical procedures rely on the accuracy of the thermodynamic, transport, and spectral properties, all of which is subject to uncertainty to a varying degree. Simplifying assumptions have been made regarding turbulence and spallation is accounted for in the form of safety margin. These assumptions need experimental verification also.

For the stagnation region where the heating rate is expected to be the highest, the question is relatively simple because there is no shear stress or turbulence. Three types of experiments have been carried out to verify the validity of the numerical techniques for this region, i.e., laser-

*Research Scientist. Member AIAA.

Assistant Chief, Thermo- and Gas-Dynamics Division. Member AIAA.

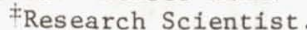

This paper is declared a work of the U.S. Government and therefore is in the public domain. irradiation, ${ }^{3,4}$ ballistic-range, ${ }^{5,6}$ and arc-jet wind tunnel tests. A gasdynamic laser was used in producing both very high-intensity (up to $1000 \mathrm{~kW} / \mathrm{cm}^{2}$ ) and moderate-intensity ( 8 to $32 \mathrm{~kW} / \mathrm{cm}^{2}$ ) radiative heat fluxes. ${ }^{3,4}$ The fluxes produced by the moderate-intensity tests are comparable to those encountered in the Jovian entry flight. In addition to determining the overall ablation rates, the particulates produced as a result of spallation were collected in the experiment. ${ }^{4}$. The mass loss due to spallation so determined varied from zero at low heating rates to about $15 \%$ of the thermochemical mass loss at $32 \mathrm{~kW} / \mathrm{cm}^{2}$ (Ref. 4). However, since boundary layer characteristicsl and spectra of incident radiation in this experiment are different from the Jovian case, the experiment was unable to test the radiative blockage phenomenon in the ablation layer which is an important parameter.

In the ballistic-range experiment, radiative heat-transfer rates slightly higher than the Jovian flight values are produced by using argon as the test gas. This experiment produced incident radiation spectra and the radiative blockage phenomenon in the boundary layer that resembled the Jovian case, but the heating history was much different: the heating was abrupt and the thermal gradient in the test material was much larger than in the Jovian flight. The results from these two experiments will be discussed in more detail later in the present work.

In the third type of experiment, ablation rates are measured in an arc-jet wind tunnel. Unlike the above two methods, this method produces boundary layer flows and heating rate histories that resemble those in Jovian flight. Moreover, spectra of radiation incident on the wall can be made identical to the Jovian case if the wind tunnel is operated with a hydrogen-helium mixture. Thermal conductivities of hydrogen and helium are both high and hence, when heated, the mixture tends to lose heat rapidly by conduction to the wall. Consequently, in order to produce temperatures sufficiently high to conduct a steady electric current, the electrical power input per unit volume must be greater than a certain critical value. A special wind tunnel, the Giant Planet Facility (GPF), was constructed at Ames Research Center in order to meet this requirement.

The Giant Planet Facility heats a 50\% hydrogen$50 \%$ helium (by volume) sixture in a constricted arc and expands it through a diverging nozzle to a low supersonic Mach number. Construction, operational characteristics, and flow diagnosis in this facility have been reported by Winovich and Carlson. ${ }^{7}$ The flow diagnosis included measurements of static pressures along the expanding nozzle, impact pressure, and total and radiative component only of the heattransfer rates to a non-ablating blunt-body calorimeter made of tungsten. Preliminary results of ablation tests in this facility and the accompanying analysis have been reported by Davy et al. ${ }^{8}$ and Green and Davy. 9

Since then, a series of comprehensive measurements has been made to determine the ablation rates 
of the two types of carbon-phenolic materials used for Galileo Probe. A graphite and a carbon-carbon composite material have been tested also for comparison. Simultaneously, spectrometric diagnosis was carried out to determine experimentally the thermodynamic state of the test flow. It is the primary purpose of the present work to report the results of these experiments and of the analytical work that accompanies them.

In the work of Green and Davy, ${ }^{9}$ the numerical prediction of ablation rates was attempted. A computer code named ARCFLO was developed to solve the problem of flow through the constricted-arc. The thermodynamic conditions of the freestream flow in the test section were determined from the pressure of the test section box and the impact pressure, assuming that enthalpy remains unchanged through the expanding nozzle. The analysis led to a Mach number value of 1.2 in the freestream. The flow-environment code RASLE and the materials-response code CMA were then run for these conditions to predict the ablation rates. The ablation rates so obtained were higher than the measured values.

The validity of the early analysis is questionable because 1) the test section box pressure is not a good indication of the true static pressure in a supersonic jet, and 2) it is not known whether the two pressure values (box and impact) are compatible with the law of momentum conservation in the expanding nozzle. It was felt necessary, therefore, to repeat the calculation with a different approach. In the present work, the process of expansion through the nozzle is calculated by integrating the momentum equation. Radial heat conduction is accounted for also in order to determine the centerline enthalpy more accurately. The expansion calculation is carried out using a code to be identified as ARCNOZ. As will be shown later, the calculation leads to a Mach number of about 2.1 instead of 1.2 . The RASLE and CMA calculations are then repeated for the new conditions. In doing so, the new sets of thermodynamic property data for $\mathrm{C}_{2} \mathrm{H}, \mathrm{C}_{3} \mathrm{H}$, and $\mathrm{C}_{4} \mathrm{H}$ are accommodated. ${ }^{10}$ For comparison with calorimeter data, RASLE calculation is made for the non-ablating case also. For comparison with the spectrometric data; the spectral intensities are calculated theoretically by carrying out a volume integration of radiation emission.

The present work collates all these experimental and theoretical results. It shows that the calculated spectra and the heat-transfer rates to the calorimeter agree closely with the experimental data. This agreement is an assurance that the flow conditions are correctly evaluated. Based on this assurance, the present work compares the experimental ablation rates with the calculated values. Only a small difference was found between the theoretical and experimental ablation rate values: the experimental values are slightly higher. The present results are then compared with those obtained by the laser-irradiation and ballistic-range experiments. Good agreement is found between the present results and the two previous results. The adequacy of the heat shield for the nose region of Galileo Probe is also discussed.

\section{Experiment}

Facility

The geometry of the wind tunnel is shown schematically in Fig. la. As shown, the arc-constrictor has a diameter of $6 \mathrm{~cm}$ and length of $432 \mathrm{~cm}$. The constrictor is followed by a divergent nozzle with a length of $33 \mathrm{~cm}$ and area ratio of 1.36 . The $50 \%$ hydrogen- $50 \%$ helium (by volume) mixture is injected into the arc-heater from the holes uniformly distributed along the constrictor length. In addition, argon is injected into the upstream end of the constrictor at a flow rate equivalent to $0.75 \%$ (by volume) of that of the hydrogen-helium mixture. The presence of argon is ignored in the present calculation. A cluster of cathodes is located at the upstream end. Four anodes are located outside the nozzle exit. Nitrogen is injected into the test section box in order to maintain a pressure of about 1 atm there. Further details of the device are described by Winovich and Carlson.?

$\underline{\text { Pressures }}$

Static pressure is measured through the pressure ports on the constrictor wall during a run. A typical measured pressure profile is shown in Fig. $1 \mathrm{~b}$ for an electrical power input of $51 \mathrm{MW}$ and test gas mass flow rate of $0.13 \mathrm{~kg} / \mathrm{sec}$. As shown, pressure falls rapidly along the axis after $\mathrm{X}=358 \mathrm{~cm}$. The $\mathrm{X}=358 \mathrm{~cm}$ point is taken to be the virtual plenum stagnation point. The measured impact pressures at the model location varied from $68 \%$ of the virtual stagnation value at lower power levels to $62 \%$ of the stagnation value at high power levels.

\section{$\underline{\text { Heat-Transfer Measurement }}$}

Heat-transfer rates at the stagnation point are determined from the temperature rise in a thin tungsten wafer forming the surface of a blunt body before it melts and evaporates. The blunt-body calorimeter is in the shape of a slightly curved disk, with a diameter of $4 \mathrm{~cm}$ and a surface radius of curvature of $20 \mathrm{~cm}$. The calorimeter normally has a shiny surface and measures the sum of the convective heat-transfer rate and the radiative rate, multiplied by the average absorptivity of the surface. The average absorptivity of the tungsten surface is determined through a separate experiment to be about 0.78 . In order to measure the radiative component of heat transfer, the calorimeter is blackened and is covered with a teflon sheath $1 \mathrm{~mm}$ thick. The transmission characteristics of the sheath are measured separately, and are corrected for in the determination of the radiative heat flux to the wall. The calorimeter is mounted on one of the four stings equipped in the test section box. Each sting is on an arm hinged at a corner of the test section box, and stays initially outside the wind tunnel flow stream. It swings into the center position after the flow is established. Figure 2 shows the results of these heat-transfer measurements.

Except for the one point at $72 \mathrm{MW}$ of input power, all data points for the total heat-transfer rate lie within a small range which indicates a good reproducibility of the experiment. The radiative component is less than half of the total heat-transfer rate. One can synthesize the true total wall heattransfer rates by first separating the total 
heat-transfer rates into convective and radiative components, dividing the radiative components by the surface absorptivity (0.78), and adding them back together. The total heat-transfer rates so determined are approximately $15 \%$ higher than the values shown.

\section{Spectrometric Measurement}

Spectrometric observation is made of the shock layer from a viewing port located at the position shown schematically in Fig. 3. Radiation emitted from the shock layer over the calorimeter, or the impact pressure probe of identical geometry as the calorimeter, is collected at one end-face of a fusedquartz fiber-optic light-pipe as shown in the figure. The other end of the light-pipe faces the entrance slit of a Tektronix J20 rapid-scanning spectrometer. In this instrument, the dispersing grating is fixed and wavelength-scan is achieved by electronically scanning over the voltage outputs from a vidicon image tube placed at the exit focal plane. The electronic signals so obtained are displayed on a storage oscilloscope. At the highest wavelength resolution used in the present work, the wavelength resolution is $0.4 \mathrm{~nm}$. One wavelength scan requires $4 \mathrm{msec}$ at the fastest scan rate.

Figures $4 a$ and $b$ show such typical data. The horizontal and vertical axes of the oscillograms correspond to wavelength and spectral intensity, respectively. Different traces indicate different scans made during the same run. Intensities of these traces differ because of the transient phenomenon. The model could have been still in the process of moving into the central position when the first scan was initiated. Or alternatively, the sensitivity of the vidicon is rising from a low initial value to the final high value: due to a peculiarity of vidicon, sensitivity is affected by the history of the average signal level, and varies slowly when the signal level changes. In either case, the cause of initially low signal is due to low radiation level of the free stream. The thermodynamic state of the shock layer flow is represented by the trace with the highest intensity.

As seen in Fig. 4a, the Balmer series lines $\mathrm{H} \gamma$ and $\mathrm{H} \delta$ at 433 and $410 \mathrm{~nm}$ of hydrogen are the only significant features in the wavelength range of 380 to $460 \mathrm{~nm}$. Hydrogen lines of shorter wavelengths, and the edge of free-bound continuum at $390 \mathrm{~nm}$ expected in this wavelength range are missing due to instrument cut-off. Grating efficiency and transmission of the fiber-optic 1ight-pipe degrade rapidly at the short wavelengths for this particular instrument setting. As mentioned in the section on Facility, argon is present in the test stream.

Argon has strong lines in the same wavelength range. Yet no argon line is seen here. This is because 1) molar concentration of argon is small compared with that of hydrogen, and 2) the excited states of argon from which the argon lines emanate are at energy levels considerably (by $2 \mathrm{ev}$ ) higher than those of hydrogen lines. In the wavelength range of 465 to $510 \mathrm{~nm}$ shown in Fig. $4 \mathrm{~b}, \mathrm{H}_{\beta}$ line is the only significant spectral feature.

\section{Flow Visualization}

An unsuccessful attempt was made to take a luminosity photograph of the shock layer flow. The region of the free-stream flow immediately ahead of the shock wave emitted such a strong radiation that no demarcation in the luminosity could be seen across the bow shock. This phenomenon is probably due to the upstream propagation of radiation, i.e., precursor phenomenon. The far ultraviolet radiation emanating from the shock layer will be absorbed by the free-stream flow ahead of the bow shock and cause electronic excitation which leads to the observed light emission.

Also unsuccessful was a laser-shadowgraph technique. The failure of this technique is attributed to the fact that electrons cause a decrease in refractive index. The increase in the index across a shock wave due to the increase in density of the neutral species may be counterbalanced by the decrease in the index due to the increase in electron density.

\section{Ablation Measurements}

Ablation measurements are made with flat-faced blunt-body models. The overall diameter of the models is $4 \mathrm{~cm}$ with the corners rounded to a radius of $0.16 \mathrm{~cm}$. The models consist of two parts: an inner core and outer shroud. The inner core is a cylindrical slug $2 \mathrm{~cm}$ in diameter, approximately $2 \mathrm{~cm}$ long. The core and the shroud are made of the same material and form a smooth flat test surface when assembled. The core is weighed prior to an ablation test. The models are mounted on the stings mentioned earlier. Two or three models are tested in this way during a single run. In some cases, the ablation tests are preceded by impact pressure or calorimeter heat-transfer rate measurements. Such instruments and ablation test models are brought into the stream only after the wind tunnel flow is fully established. The ablation models are brought out of the test stream after a preprogrammed time interval. The duration of the ablation tests is varied between 1 and $7 \mathrm{sec}$. After the test, the core slug is weighed to determine the change in mass. By dividing the mass change by the wet surface area, mass loss per unit area $\Delta \mathrm{m}$, in $\mathrm{g} / \mathrm{cm}^{2}$, is obtained. Geometrical recession is measured also to compare with the mass loss data. Recession and mass loss are found to be related consistently through the average density of the material.

The ablation was conducted for 18 ATJS graphite models, 3 multidimensional carbon-carbon composite models, 3 chop-molded carbon-phenolic models with type FM5055A prepreg, 3 chop-molded carbon-phenolic models with type FM5055G prepreg, 3 tape-wrapped carbon-phenolic models with type FM5055A prepreg, and 3 tape-wrapped carbon-phenolic models with type FM5055G prepreg. The chop-molded and tape-wrapped carbon-phenolic with FM5055G prepreg are the same materials as those used on Galileo Probe. In testing the tape-wrapped carbon-phenolic, the planes of the tape cloths were at $30^{\circ}$ to the ablating surface as they are on Galileo Probe.

The ablation tests are conducted at a fixed wind tunnel condition of electrical power input of $71 \mathrm{MW}$ on the average and test gas mass flow rate of $0.160 \mathrm{~kg} / \mathrm{sec}$. In Figs. 5 and 6 , the mass 1 oss $\Delta \mathrm{m}$ obtained from the tests is plotted against the test duration. As seen in the figures, mass losses are reproducible and consistent. Recession occurs smoothly with time. The posttest examination revealed a smooth ablated surface for all tested models. Surface roughness was no more than the intrinsic roughness of the fibrous structure of the materials. No sign of massive spallation was seen. 


\section{Theory}

\section{Calculation of Flow Conditions}

As mentioned in Introduction, the code ARCFLO was used in calculating the flow through the arcconstrictor. ${ }^{9}$ This code rigorously applies mass and energy conservation laws, but fails to apply the momentum conservation law. This is because 1) momentum balance is dictated strongly by turbulence which is not well understood, and 2) the code is numerically parabolic with respect to axial distance, and hence cannot accommodate pressure feedback occurring in a subsonic flow. Because of these limitations, the code cannot accommodate the total wall injection of the test gas. The closest to the real case is obtained under the assumption that $50 \%$ of the test gas is introduced in the upstream end and the remainder of the gas from the wall. A best agreement between the calculated and measured arc-heater performance was found with the assumption that the effective turbulent viscosity and thermal conductivity are three times their laminar values. This solution will be referred to as the reference ARCFLO solution.

General features of the reference ARCFLO solution are listed in Table 1. The solution shows that rapid changes in flow properties in the radial direction occur within the outermost $4 \%$ of the constrictor radius containing laminar sublayer. The inner $96 \%$ of the radius is occupied by a turbulent core flow in which properties change gradually. The pressures obtained by the solution, shown in Fig. 1b, agree with experiment in the first $2 / 3$ of the length of the constrictor. In the downstream $1 / 3$, the calculated pressures are higher than the measured values because of the above-mentioned limitations.

In order to determine the flow conditions at the model location one needs a code which applies momentum balance in the expanding region correctly, and joins with the ARCFLO solution at throat with an appropriate correction for pressure there. Such a code needs to be accurate only in the centerline region of the flow where the model is located. A code named ARCNOZ is developed for this purpose under the following assumptions:

1) Pressure is constant in the radial direction and is prescribed in the axial direction.

2) Electrical power input and radiative power loss are equal and cancel each other.

3) Wall is adiabatic and frictionless.

4) Nozzle divergence angle is so small that cosine of the angle can be taken as 1 .

Assumption 2) is derived from the observation that, both theoretically and experimentally, electrical power input nearly equals the heat loss to the wall in the downstream portion of the constricted-arc flow. Assumption 3) is made because, over the short distance of expansion, the wall heat transfer and wall friction cannot influence the conditions in the centerline region. Under this assumption, slopes are zero at wall for both velocity and enthalpy profiles, which simplifies analysis greatly.

The equations to be solved are

$$
\rho U \frac{\partial U}{\partial x}=-\frac{d P}{d x}+\frac{1}{r} \frac{\partial}{\partial r}\left(r \mu \frac{\partial u}{\partial r}\right)
$$

$$
\rho U \frac{\partial h}{\partial x}=U \frac{d p}{d x}+\frac{1}{r} \frac{\partial}{\partial r}\left[r k \frac{\partial T}{\partial r}+\frac{r}{2}\left(\mu-\frac{k}{C_{p}}\right) \frac{\partial U^{2}}{\partial r}\right]
$$

where $\rho, U, p, h, T$, and $C_{p}$ are density, axial velocity, pressure, static enthalpy, temperature, and specific heat at constant pressure, respectively. The quantities $\rho$ and $k$ are viscosity and thermal conductivity, respectively, which are taken to be three times the laminar values as are for ARCFLO. The flow is assumed to be in equilibrium. Pressure variation along the axis is assumed to occur as if the test gas was perfect with $\gamma=C_{p} / C_{v}=1.4$ and passed through a hyperbolic nozzle of cross-sectional area A described by $A / A^{*}=1+B X^{2}, A^{*}$ being the throat area. Figure la shows the profile of such a nozzle. Figure $1 \mathrm{~b}$ shows a pressure profile corresponding to such a nozzle. Rankine-Hugoniot relationship and the subsonic compression process behind the shock are solved in order to calculate the impact pressure at the centerline. The parameter $B$ in the area ratio specification is varied arbitrarily until the calculated impact pressure agrees with the measured value.

The calculation requires velocity and enthalpy profiles at throat as inputs. The electrical power input, mass flow rate, and pressure at throat for the experimental conditions are different from those of reference ARCFLO solution. The initial profiles for the experimental conditions are derived from the ARCFLO solution under the assumption that velocity and total enthalpy profiles at throat are similar between the theoretical and the experimental cases. Under this assumption, the experimental profiles can be obtained by multiplying the ARCFLO profiles by the following correction factors at each radial point:

Experimental total enthalpy ARCFLO total enthalpy

= Experimental power input ARCFLO mass flow rate ARCFLO power input Experimental mass flow rate

Experimental velocity ARCFLO velocity

$=\frac{\text { ARCFLO pressure }}{\text { Experimental pressure }} \frac{\text { Experimental mass flow rate }}{\text { ARCFLO mass flow rate }}$

The equations are solved numerically. In Fig. 7, the resulting radial variation of static enthalpy, temperature, and velocity are shown at throat and at the test section for the ablation test conditions. In addition, the conditions behind a normal shock wave are shown for the centerline region. As shown in the figure, the virtual wall radius changes from $3 \mathrm{~cm}$ at throat to $4.93 \mathrm{~cm}$ at the test section, corresponding to an area ratio of 2.7 . This is substantially larger than the geometrical area ratio of 1.36 , and is believed to be due to the nonuniform radial distribution of properties seen in the figure. The nonuniformity produces a large displacement thickness at throat and reduces the effective cross-sectional area there.

The ARCNOZ solution is obtained for Runs 201 and 219 referred to in Fig. 4 and for the average conditions of the runs made for the ablation measurement. The results are summarized in Table 1 . In the table, the static pressure of the free stream 
in the test section is lower than the pressure in the test section box. This condition is possible because a portion of the dynamic pressure of the jet flow can be converted to static pressure: only about $1 / 3$ of the $\mathrm{pU}^{2}$ in the jet needs to be converted to static pressure in order for these two pressures to balance. The centerline total enthalpy in the test section is about $94 \%$ of that at throat. This difference is due to turbulent conduction of heat from the centerline to the outer region. As a result of this conduction, the ratio of centerline to average static enthalpies decreases from 2.1 at throat to 1.5 at test section. Mach number at the centerline of the test section is between 2 and 2.2.

As seen in Fig. 7, flow properties vary across the test section. As will be described later, the present ablation rate tests measure the average values over radial distances of up to $1.0 \mathrm{~cm}$. Radiative heat fluxes incident on any point on a flat surface is an integration of radiant intensity over an incident angle. It is known in the radiative transfer theories that the largest contribution to the radiative heat flux comes from the $45^{\circ}$ direction. Such reasoning leads to the belief that, for the calculation of convective and radiative heattransfer rates to the stagnation region in the present experiment, the averages of the flow properties in the range of radius of 0 to $1.5 \mathrm{~cm}$ must be used. Table 1 , therefore, lists the average properties in the free stream over this range. For the ablation test conditions, the average properties are: $0 \cong 0.00101 \mathrm{~kg} / \mathrm{m}^{3}, \mathrm{U} \cong 15.8 \mathrm{~km} / \mathrm{sec}$, $\mathrm{h} \cong 260 \mathrm{MJ} / \mathrm{kg}$, and total enthalpy $\mathrm{h}+(1 / 2) \mathrm{U}^{2}=380 \mathrm{MJ} / \mathrm{kg}$. Approximately $1 / 3$ of the total enthalpy is in the kinetic form.

\section{Interpretation of Spectral Data}

Shock stand-off distance, which controls radiative heat-transfer rate, and the radial velocity gradient which controls convective heat-transfer rate, are both related to the curvature of the bow shock wave. For an arbitrarily shaped blunt body, an equivalent-hemisphere can be defined that corresponds to the actual curvature of the shock wave. For a flat disk, this relationship is expressed in terms of the average density ratio across the shock $\varepsilon$, i.e., the ratio between the free-stream density and the average density between the aftershock point and the (inviscid) stagnation point. According to Park, ${ }^{11}$ the equivalent nose radius $R_{n}$ is related to the disk radius $R_{d}$ by

$$
\frac{R_{n}}{R_{d}}=\frac{1+\varepsilon}{\sqrt{\varepsilon}\left(1-2 \varepsilon+5.5 \varepsilon^{2}\right)}
$$

The disk radius is taken to be $1.9 \mathrm{~cm}$ in the present work. Shock stand-off distance $\Delta$ is given by

$$
\frac{\Delta}{\mathrm{R}_{\mathrm{d}}}=\sqrt{\varepsilon}(1+\varepsilon)
$$

Shock radius is obtained by adding the shock standoff distance to the nose radius. For the conditions of ablation tests in the present work, these parameters were $\varepsilon=0.210, R_{n}=6.09, \Delta=1.06 \mathrm{~cm}$, and $\mathrm{R}_{\mathrm{S}}=7.15 \mathrm{~cm}$, respectively.

As mentioned earlier, the calorimeter model had a surface radius of curvature of $20 \mathrm{~cm}$. The change in shock curvature due to this surface curvature is calculated from the mass conservation relationship. One first derives the expression for the "new" shock stand-off distance produced as a result of the surface curvature. The expression contains the new unknown shock radius as a parameter. Separately, the change in radial acceleration is expressed in terms of the new shock radius. One then invokes the mass conservation law which requires that the fractional change in radial acceleration equals in magnitude the fractional charge in shock stand-off distance and is opposite in sign. By solving for the new shock radius $R_{S}^{\prime}$, one obtains:

$$
\frac{R_{s}^{\prime}}{R_{s}}=1-\frac{R_{d}}{\Delta} \frac{R_{d}}{R_{f}}\left\{1+\frac{R_{s}}{\Delta}\left[\sqrt{1-\left(R_{d} / R_{s}\right)^{2}}-1\right]\right\}^{-1}
$$

where $R_{f}$ is the radius of curvature of the model surface. For the present case, one has $\mathrm{R}_{\mathrm{S}}^{\prime} / \mathrm{R}_{\mathrm{S}}=0.956$. The equivalent nose radius and shock stand-off distance for the calorimeter are $\mathrm{R}_{\mathrm{n}}^{\prime}=5.82 \mathrm{~cm}$ and $\Delta^{\prime}=1.01 \mathrm{~cm}$, respectively.

The relative radiation spectra emitted by the shock layer flow and received by the spectrometer are calculated for the wavelength range of 390 to $500 \mathrm{~nm}$ for the purpose of comparison with the experimental data of Fig. 4. The calculation is made using the following methods:

1) The radiation is assumed to be optically thin. This assumption is derived through a detailed examination of the optical properties of the shock layer and the flow in the test section box.

2) For the volume integration of the emissions from different parts of the shock layer, the shock layer is divided into 9 concentric cylindrical ring elements of $0.246 \mathrm{~cm}$ thickness. The rings extend from the bow shock to the wall, the coordinates of which are obtained from the known shock and body geometry. The properties are assumed to be constant within each ring, and are determined by averaging between the after-shock and the (inviscid) stagnation values. Radiation from each ring is added and weighted with its volume to obtain the total radiation entering the spectrometer.

3) Radiation emanating from the small volume of the free stream in front of the shock layer is accounted for in the same manner. The length of the emitting cylinder is assumed to be $1 \mathrm{~cm}$ in this case.

4) The calculation is carried out using the line-by-line radiative transfer code ARCRAP, accounting for the lines and continua of hydrogen. ${ }^{2}$

The results of these calculations are shown in Figs. $8 a$ and $b$, and are compared with the experimental data. The agreement is generally good. In Fig. 8a, the experimental data are not shown for wavelengths shorter than $408 \mathrm{~nm}$, because experimental data are not accurate there (see Spectrometric Measurement). The small peak in intensity at $485 \mathrm{~nm}$ in Fig. $8 \mathrm{~b}$ is believed to be due to $\mathrm{N}_{2}{ }^{+}$firstnegative band radiation. As mentioned earlier (see Facility), nitrogen is injected into the test section box. The gas is probably entrained by the jet, heated, and emits the observed radiation. The small discrepancy in the peak intensity of $\mathrm{H} \delta$ line at $410 \mathrm{~nm}$ in Fig. $8 \mathrm{a}$ is due to the inadequacy of the computational method in this wavelength region in calculating the merging of the highly excited states 
of hydrogen. The shapes of $\mathrm{H}_{\beta}$ and $\mathrm{H}_{\gamma}$ lines are the most reliable because the theoretical shapes of these lines are accurately known. The good agreement between the measured and calculated line profiles for these two lines is a verification of the validity of the flow field model used in the present work.

\section{Heat Transfer and Ablation}

The flow in the stagnation region over the two geometries of the present experiment (curved disk for calorimeter and flat disk for ablation tests) are solved using the code RASLE. The solutions are obtained for the free-stream conditions identified as Ablation Test case in Table 1 . The thermodynamic data for $\mathrm{C}_{2} \mathrm{H}, \mathrm{C}_{3} \mathrm{H}$, and $\mathrm{C}_{4} \mathrm{H}$ (Ref. 10), and the radiation absorption by $\mathrm{C}_{3}$ in the ultraviolet wavelength range ${ }^{13,14}$ are accounted for in the calculation. The calculation is carried out for four cases: the nonablating tungsten, nonablating carbon (graphite and carbon-carbon) or carbon-phenolic, carbon in steady-state ablation, and carbon-phenolic in steadystate ablation. The shock radii given in the preceding section are given as inputs. For the case of tungsten, surface temperature and spectral emissivity are the same as in the work of Green and Davy. ${ }^{9}$ For carbon and carbon-phenolic, surface spectral emissivity is assumed to be constant at 0.85 . The results of these calculations are summarized in Table 2. The heat-transfer rates for the tungsten case are plotted in Fig. 2 and compared with the measured data.

In Fig. 2, the straight dash lines are shown for reference only. As the figure shows, the calculated heat-transfer rate values are consistent with the measured values. As mentioned earlier, the experimental total heat-transfer rates include only $78 \%$ of the radiative component. When correction is made to account for this fact, agreement between theory and experiment is good. The agreement is a further verification of the validity of the present flow model. Radiative heat-transfer rate comprises about $1 / 3$ of the total heat-transfer rate in this nonablating case.

Under the steady-state ablating conditions, however, convective heat-transfer rates decrease by an order of magnitude from the nonablating values because of the convective shielding effect of blowing. The blowing parameter $B^{\prime}=\dot{m} /\left(\rho U C_{h}\right)$, where $\dot{\mathrm{m}}$ is the ablation rate and $\mathrm{C}_{\mathrm{h}}$ is the heat-transfer coefficient, reaches moderate values of 6 and 14 . As a result, the convective component of heat flux is negligible at the wall. Due to absorption by the ablation-product gases in the blowing layer, the radiative heat fluxes reaching the wall is roughly $3 / 4$ of those incident on the interface between the inviscid and the blowing layers. These phenomena are qualitatively the same as those expected to occur during the Jovian entry flight. However, the absolute magnitudes of heat-transfer and ablation rate are considerably smaller than the peak values encountered in the Jovian flight.

For carbon and carbon-phenolic, ablation behavior is calculated using the CMA code. The properties of the two materials are taken to be the same as those used in the work of Park. ${ }^{5,6}$ Radiative blockage factor (the ratio of the radiative heat flux reaching wall to that at the interface) is assumed to vary as
Radiative blockage factor $=\exp (-\mathrm{C} \dot{\mathrm{m}})$

This relationship is a special case of Beer's law of radiation absorption, and holds rigorously if the optical depth of the blowing layer is proportional to ablation rate. The constant $C$ in the above equation is chosen so as to be compatible with the RASLE solutions obtained. The convectiveshielding factor is calculated using the well-known formula. ${ }^{15}$ The heat-transfer rates so determined are then given as inputs to the CMA code.

The results of the CMA calculations are presented in Figs. 5 and 6 , and are compared with the experimental data. For all tested materials, the measured mass loss rates are about $15 \%$ higher than the theoretical values. This discrepancy may be attributed to spallation. The carbon-phenolic materials show about $25 \%$ higher ablation rates than either graphite or carbon-carbon. This difference in ablation performance between the two groups of materials is well within the common expectations of the materials.

\section{$\underline{\text { Discussion }}$}

The good agreement between theory and experiment in spectral data (Figs. $8 \mathrm{a}$ and b) and heattransfer rates (Fig. 2) provides confidence in the present method of evaluation of ablative performance of the tested materials. The small discrepancy between the theoretical and experimental ablation rates are attributed presently to spallation. As mentioned earlier, the ablating surface of the models were fairly smooth after test. It is not known how spallation occurs without leaving a roughened surface, but such a phenomenon has been known to exist for graphite, ${ }^{16}$ and hence is not new.

In Fig. 9, the present ablation data for carbon-phenolic are compared with the two other sets of data taken under radiative heating environments, i.e., the laser-irradiation ${ }^{3,4}$ and ballisticrange ${ }^{5,6}$ data. The two dash lines in the figure correspond to the effective heats of vaporization of $22 \mathrm{MJ} / \mathrm{kg}$ used in the earlier calculations of Galileo Probe heat shield, and $30 \mathrm{MJ} / \mathrm{kg}$ resulting from the present thermodynamic model. The ballistic-range data were interpreted using the same thermodynamic model, which leads to a vaporization energy value of $31 \mathrm{MJ} / \mathrm{kg}$ in the high pressure environment of the experiment. The laser results obtained at heat fluxes higher than $41 \mathrm{~kW} / \mathrm{cm}^{2}$ (Ref. 3) are not compared here because the data are subject to an uncertainty as to how much of the laser power reached the wall of the test specimen: at such high heat fluxes, the rate of spallation is so great that the spalled particles partly block the oncoming laser beam. The ballistic-range values shown in the figure are obtained by multiplying the theoretical recession rates ${ }^{5}$ by the ratio of the overall measured recession to the theoretical recession. ${ }^{6}$

As the figure shows, the three sets of data are consistent and form a continuous trend. At low wall heat fluxes, the measured ablation rates follow the $30 \mathrm{MJ} / \mathrm{kg}$ reference line. At high heat fluxes, the measured ablation rates approach the $22 \mathrm{MJ} / \mathrm{kg}$ line. The upward trend is attributed to spallation. The observed agreement at low heating rates lends support to the vaporization energy value of $30 \mathrm{MJ} / \mathrm{kg}$ and the associated new thermodynamic data for $\mathrm{C}_{2} \mathrm{H}$, $\mathrm{C}_{3} \mathrm{H}$, and $\mathrm{C}_{4} \mathrm{H}$ used here. ${ }^{10}$ Based on the heat of 
vaporization value of $30 \mathrm{MJ} / \mathrm{kg}$, spallation rate is about $15 \%$ of the total ablation rate at the wal1 heat fluxes of about $20 \mathrm{~kW} / \mathrm{cm}^{2}$ and about $30 \%$ at fluxes above $30 \mathrm{~kW} / \mathrm{cm}^{2}$.

The consistency among the three sets of data is an indication of the validity of the three experiments. It was shown earlier that the thickness of the heat shield on Galileo Probe contains sufficient margin of safety to allow for the spallation of the magnitudes observed here. ${ }^{4}$ The present test results reinforce that conclusion.

\section{Conclusions}

An ATJS graphite, a carbon-carbon composite, and four types of carbon-phenolic materials are heated in the stagnation region of a blunt body in an arc-jet wind tunnel stream of hydrogen-helium mixture. The tested conditions are: total enthalpy $\cong 380 \mathrm{MJ} / \mathrm{kg}$, impact pressure $\cong 3 \mathrm{~atm}$, and Mach number $\cong 2.1$. The wall heating is almost entirely by radiation, and its rate is about $6 \mathrm{~kW} / \mathrm{cm}^{2}$. Compared with the theoretical predictions made using RASLE and CMA codes, the tested materials ablate at a rate about $15 \%$ faster. The measured ablation rates agree with those obtained in a laserirradiation facility and in a ballistic-range.

\section{References}

${ }^{1}$ Howe, J. T., Pitts, W. C., and Lundell, J. H., "Survey of the Supporting Research and Technology for Thermal Protection of the Galileo Probe," AIAA Paper 81-1068, Palo Alto, Calif., June 1981.

${ }^{2}$ Menees, G. P., "An Evaluation of Computer Codes for Simulating the Galileo Probe Aerothermal Entry environment," AIAA Paper 81-1069, Palo Alto, Calif., June 1981 .

${ }^{3}$ Lunde11, J. H. and Dickey, R. R., "Response of Heat-Shield Materials to Intense Laser Radiation," Progress in Astronautics and Aeronautics: Outer Planet Entry Heating and Thermal Protection, Vol. 64, edited by R. Viskanta, AIAA, New York, 1979, pp. 193-209.

${ }^{4}$ Lunde11, J. H., "Spallation of the Galileo Probe Heat Shield," AIAA Paper 82-0852, St. Louis, Missouri, June 1982.

${ }^{5}$ Park, C., "Stagnation-Point Ablation of Carbonaceous Flat Disks: Part 1. Theory," accepted for publication in AIAA Journal.

${ }^{6}$ Park, C., "Stagnation-Point Ablation of Carbonaceous Flat Disks: Part 2. Experiment," accepted for publication in AIAA Journal.
${ }^{7}$ Winovich, W. and Carlson, W. C. A., "The Giant Planet Facility," Paper presented at 25th Instrument Society of American Symposium, Los Angeles, April 1979.

${ }^{8}$ Davy, W. C., Menees, G. P., Lundel1, J. H., and Dickey, R. R., "Hydrogen-Helium Ablation of Carbonaceous Materials: Numerical Simulation and Experiment," Progress in Astronautics and Aeronautics: Outer Planet Entry Heating and Thermal Protection, Vo1. 64, R. Viskanta, Ed., AIAA, New York, 1979, pp. 228-244.

${ }^{9} \mathrm{Green}$, M. J. and Davy, W. C., "Numerical Simulation of Experiments in the Giant Planet Facility," Progress in Astronautics and Aeronautics: Entry Heating and Thermal Protection, Vol. 69, W. B. O1stad, Ed., AIAA, New York, 1980, pp. 172-197.

${ }^{10}$ Moss, J. N. and Simmonds, A. L., "Galileo Probe Forebody Flow Field Predictions During Jupiter Entry," AIAA Paper 82-0874, St. Louis, Missouri, June 1982 .

${ }^{1}$ Park, C., "Calculation of Radiation From Argon Shock Layers," Journal of Quantitative Spectroscopy and Radiative Transfer, Vol. 28, No. 1, July 1982, pp. 29-40.

${ }^{12}$ Arnold, J. 0., Cooper, D. M., Park, C., and Prakash, S. G., "Line-by-Line Transport Calculations for Jupiter Entry Probes," Progress in Astronautics and Aeronautics: Entry Heating and Thermal Protection, Vol. 69, W. B. O1stad, Ed., AIAA, New York, 1980 , pp. 52-82.

${ }^{13}$ Prakash, S. G. and Park, C., "Shock Tube Spectroscopy of $\mathrm{C}_{3}+\mathrm{C}_{2} \mathrm{H}$ Mixture in the 140-700 nm Range," AIAA Paper 79-0094, New Orleans, Louisiana, January 1979.

${ }^{14}$ Shinn, J. L., "Optical Absorption of Carbon and Hydrocarbon Species from Shock Heated Acetylene and Methane in the 135-200 nm Wavelength Range," AIAA Paper 81-1189, Palo Alto, Calif., June 1981.

${ }^{15}$ Dorrance, W. H., Viscous Hypersonic Flow, McGraw-Hi11, New York, N.Y., 1962, pp. 112-114.

${ }^{16}$ Lunde11, J. H., and Dickey, R. R., "Ablation of ATJ Graphite at High Temperatures," AIAA Journal, Vol. 11, No. 2, February 1973, pp. 216-222. 
Table 1 Measured (asterisked) and Calculated Flow Properties

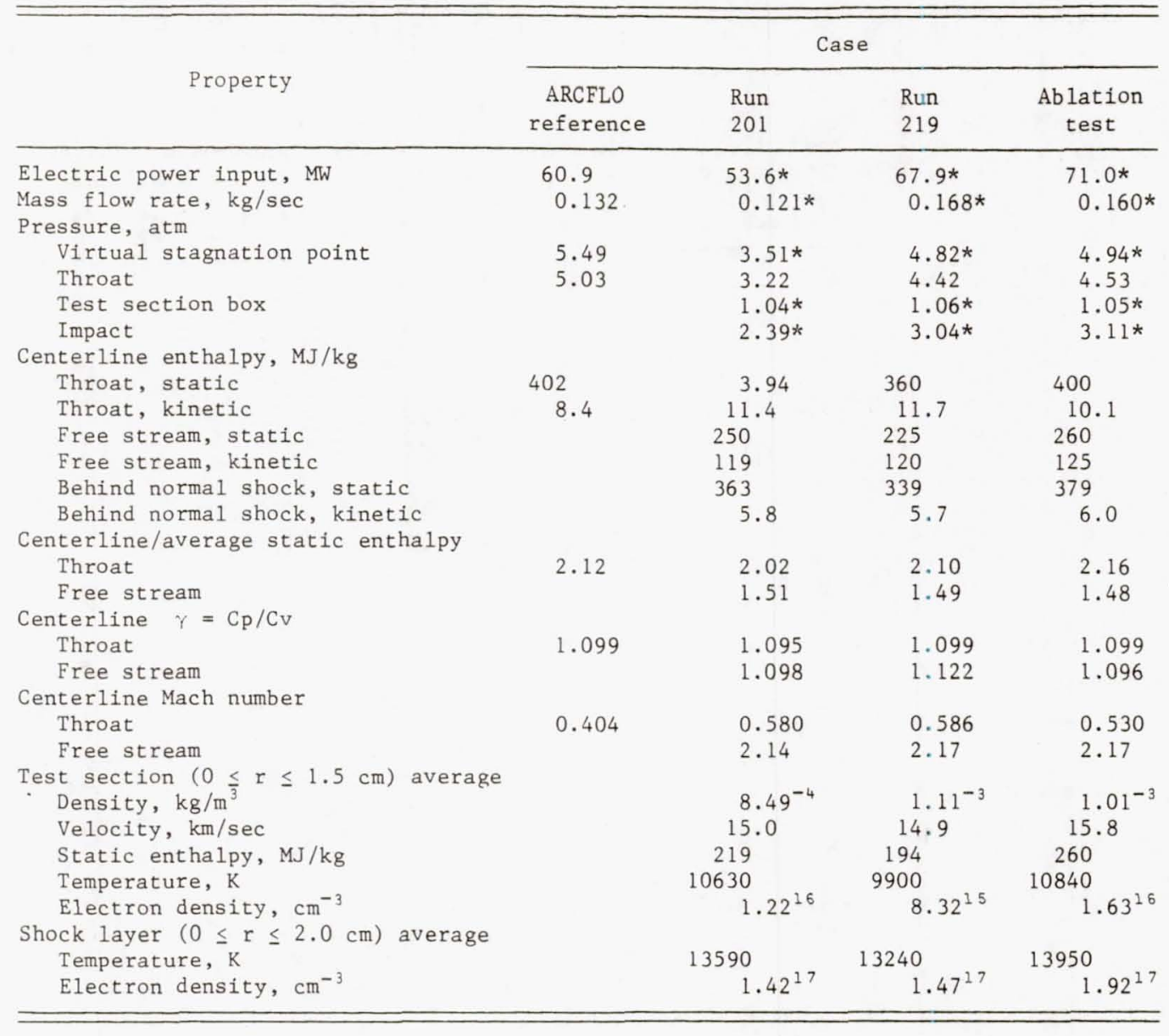

Table 2 RASLE Solutions for Ablation Tests (Input Power $=71 \mathrm{MW}$ )

\begin{tabular}{|c|c|c|c|c|c|}
\hline & \multirow{2}{*}{$\begin{array}{c}\begin{array}{c}\text { Tungsten } \\
\text { calorimeter }\end{array} \\
\begin{array}{c}\text { No } \\
\text { blowing }\end{array}\end{array}$} & \multicolumn{2}{|c|}{$\begin{array}{l}\text { Graphite and } \\
\text { carbon-carbon }\end{array}$} & \multicolumn{2}{|c|}{ Carbon-phenolic } \\
\hline & & $\begin{array}{c}\text { No } \\
\text { blowing }\end{array}$ & $\begin{array}{l}\text { Steady- } \\
\text { state }\end{array}$ & $\begin{array}{c}\text { No } \\
\text { blowing }\end{array}$ & $\begin{array}{l}\text { Steady- } \\
\text { state }\end{array}$ \\
\hline Radiative heat flux at interface, $\mathrm{KW} / \mathrm{cm}^{2}$ & 6.76 & 6.98 & 7.39 & 6.98 & 7.34 \\
\hline Radiative heat flux at wall, $\mathrm{KW} / \mathrm{cm}^{2}$ & 6.61 & 6.85 & 4.94 & 6.85 & 5.79 \\
\hline Wall convective heat flux, $\mathrm{KW} / \mathrm{cm}^{2}$ & 13.9 & 11.8 & 0.646 & 11.8 & 0.327 \\
\hline Wa11 temperature, $\mathrm{K}$ & 2000 & 4094 & 4278 & 4094 & 4203 \\
\hline Ablation rate, $\dot{\mathrm{m}}, \mathrm{g} / \mathrm{cm}^{2}-\mathrm{sec}$ & 0 & 0 & 0.154 & 0 & 0.170 \\
\hline Blowing parameter, $B^{\prime}=\dot{m} /\left(\rho_{\infty} V_{\infty} C_{n}\right)$ & 0 & 0 & 8.65 & 0 & 18.7 \\
\hline Radiative blockage factor & 0.978 & 0.981 & 0.668 & 0.981 & 0.788 \\
\hline Convective shielding factor & 1.0 & 1.0 & 0.0547 & 1.0 & 0.0277 \\
\hline
\end{tabular}




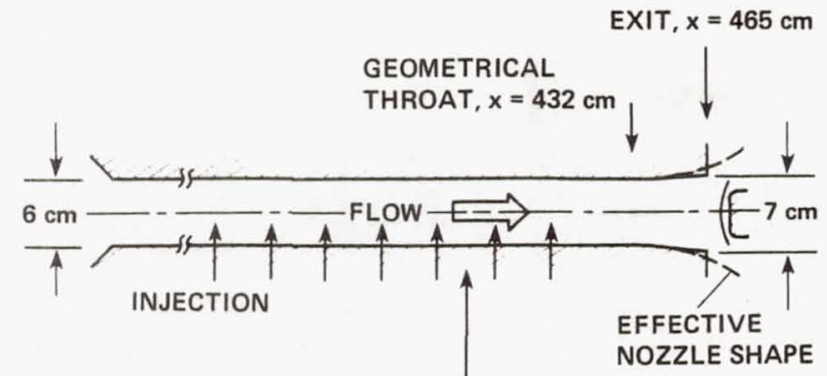

VIRTUAL PLENUM STAGN. POINT, $x=358 \mathrm{~cm}$

(a) GPF GEOMETRY

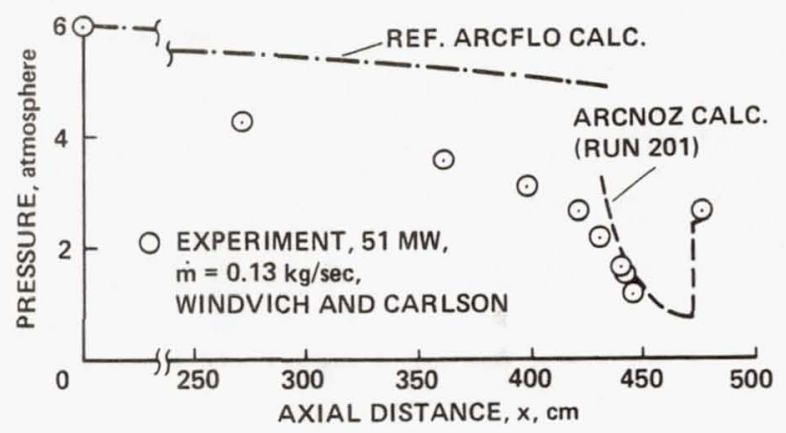

(b) PRESSURE VARIATION

Fig. I Geometry of Giant Planet Facility and pressure variation.

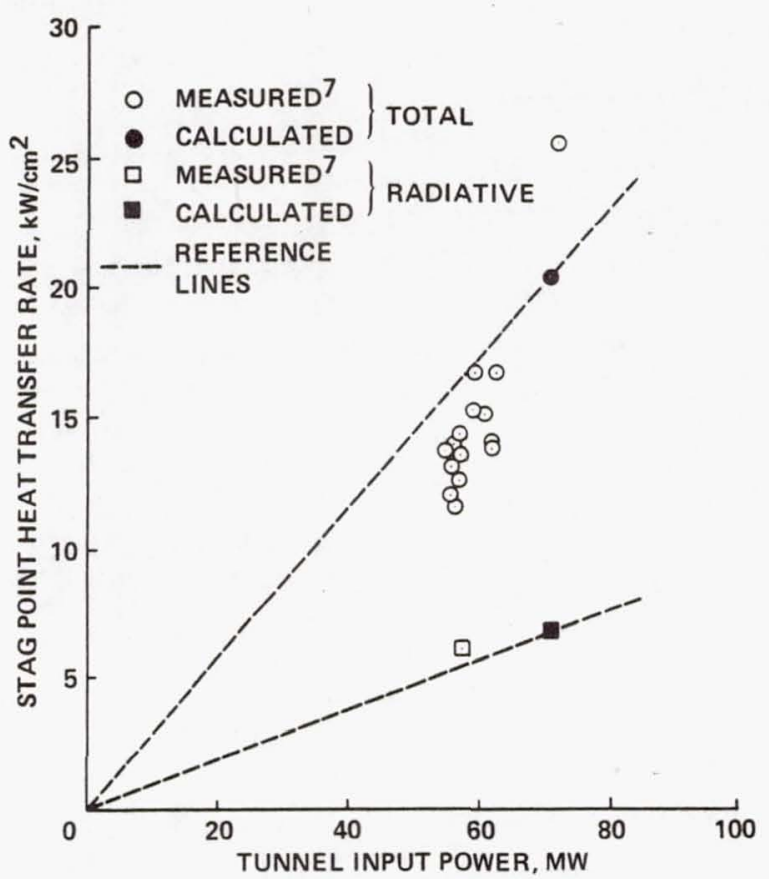

Fig. 2 Comparison between the measured and calculated heat transfer rates to the nonablating calorimeter. The experimental data are taken at mass flow rates between 0.12 and $0.17 \mathrm{~kg} / \mathrm{sec}$. The theoretical points are for the mass flow rate of $0.16 \mathrm{~kg} / \mathrm{sec}$.

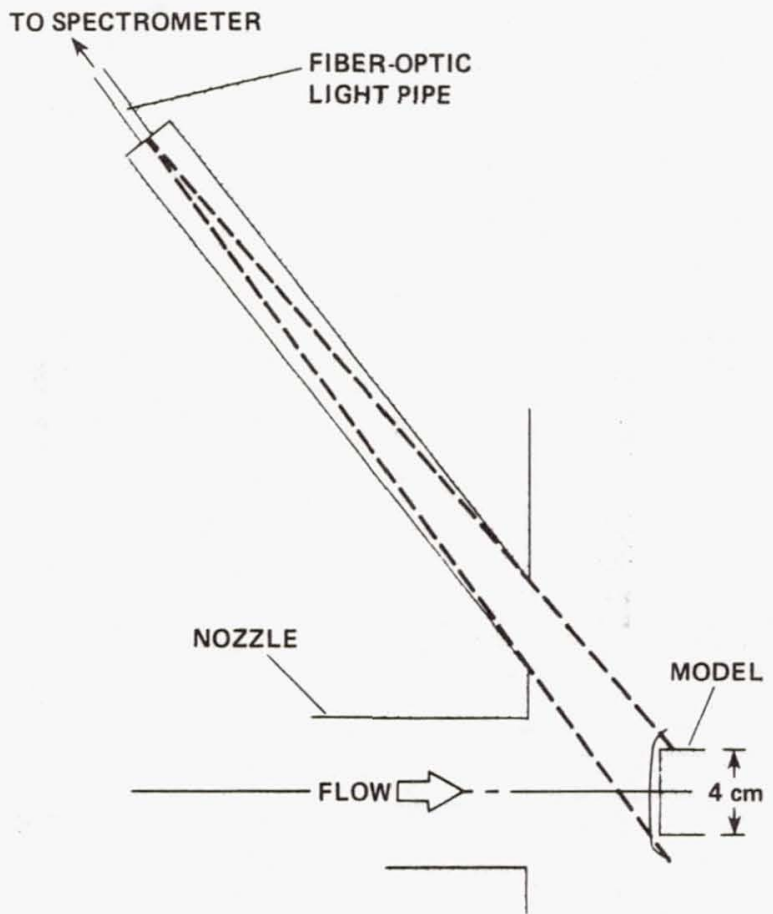

Fig. 3 Optical layout of spectroscopic measurement. 


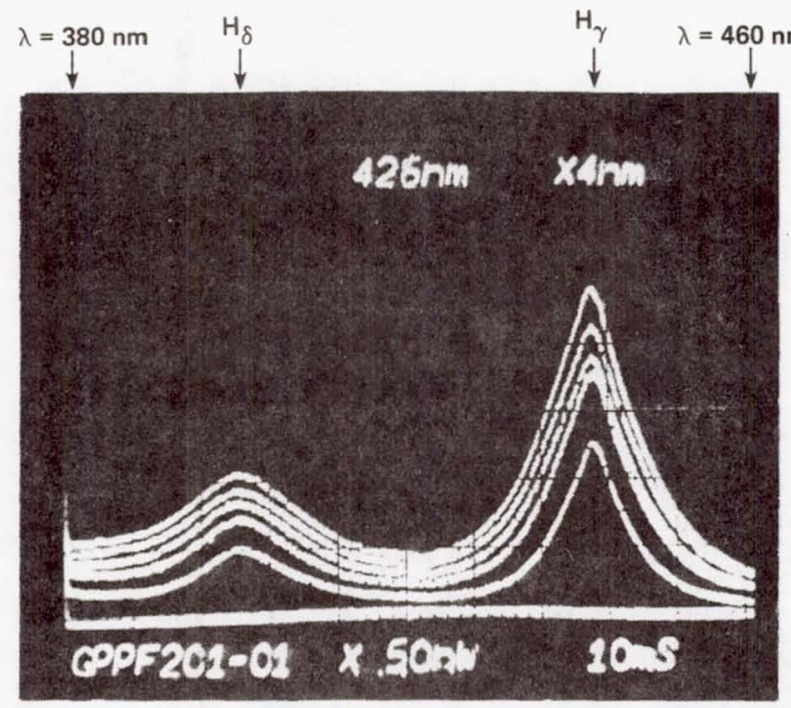

(a) $380<\lambda<460 \mathrm{~nm}$, RUN 201

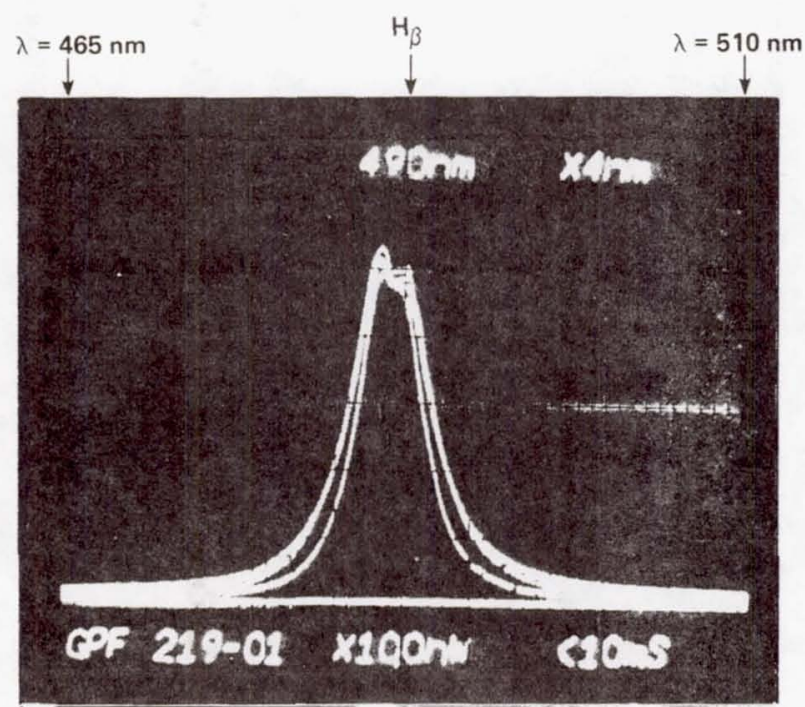

(b) $465<\lambda<510 \mathrm{~nm}$, RUN 219

Fig. 4 Oscillogram record of spectral intensities of shock layer radiation.

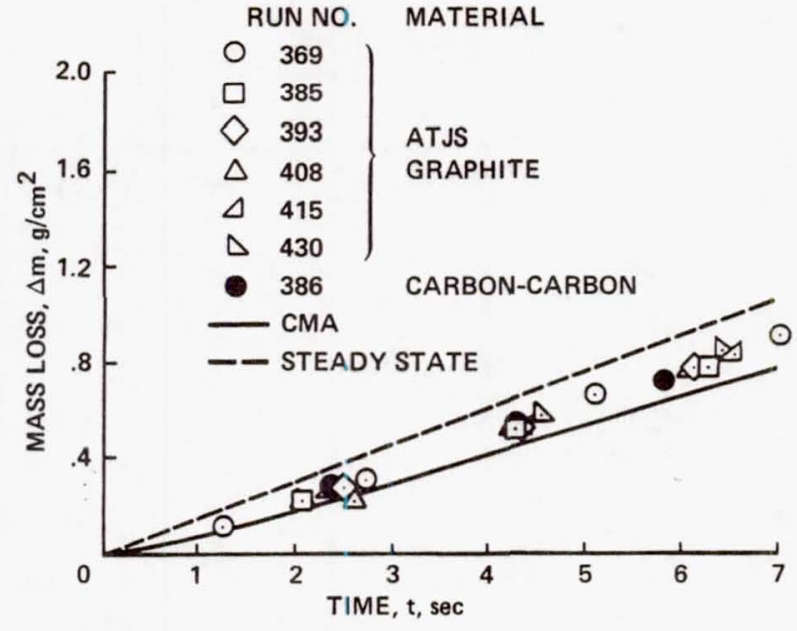

Fig. 5 Comparison between theory and experiment on mass loss rate for ATJS graphite and carbon-carbon composite models.

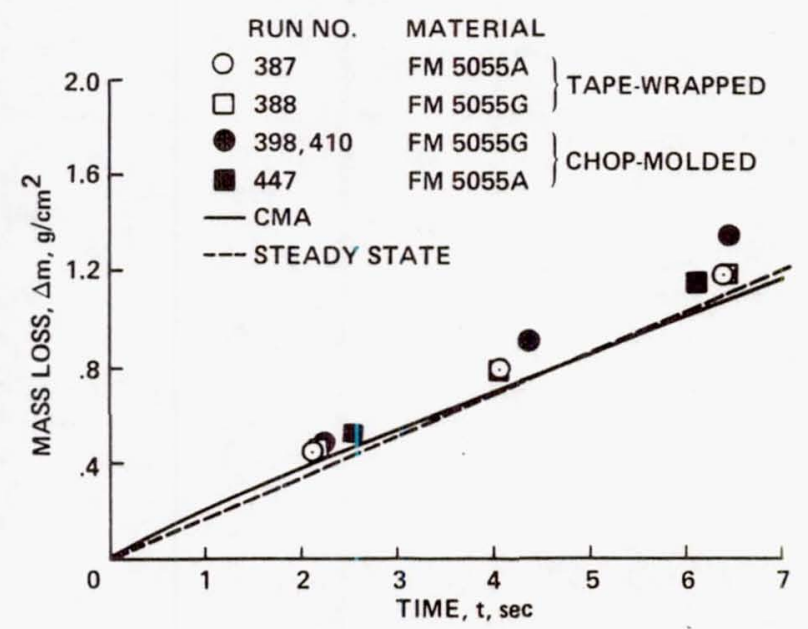

Fig. 6 Comparison between theory and experiment on mass loss rate for carbon-phenolic models. 

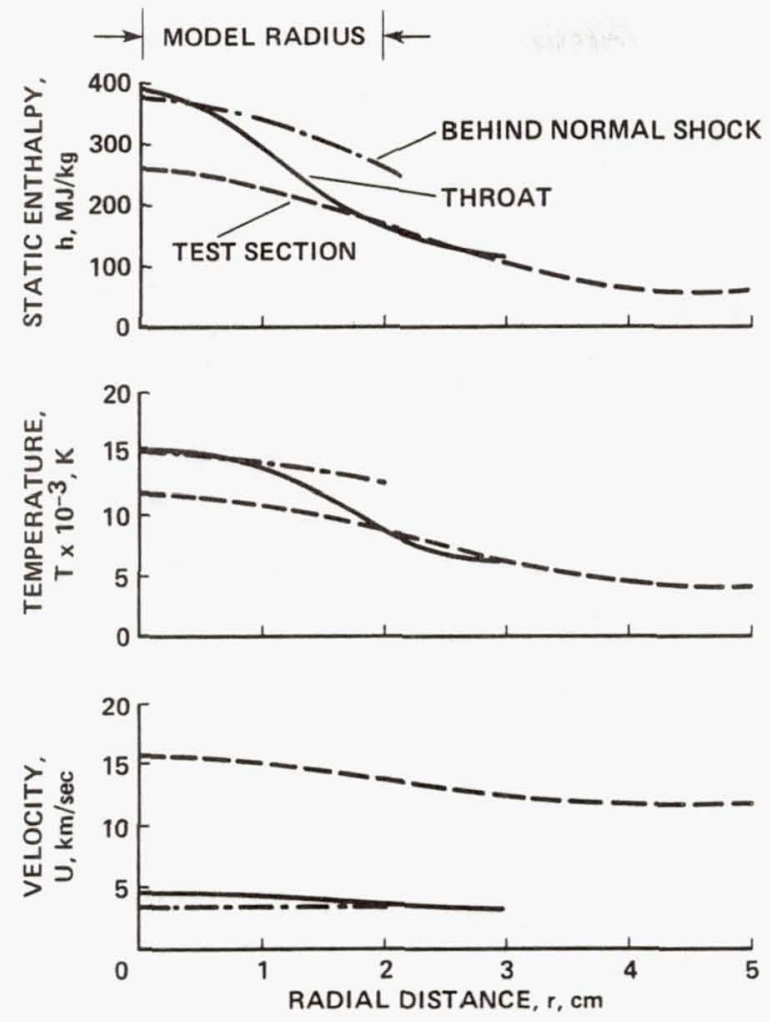

Fig. 7 ARNOZ solution for the ablation test condition.

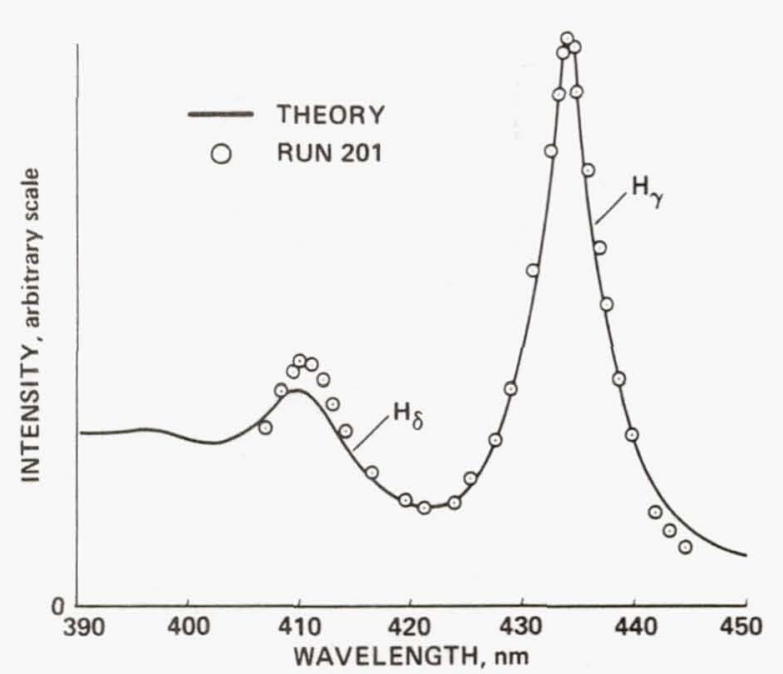

(a) Run 201.

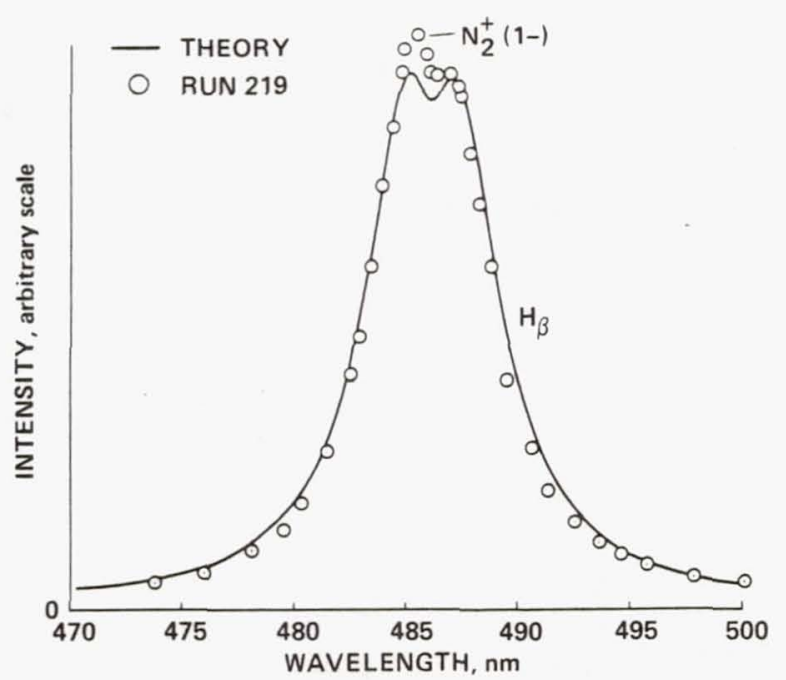

(b) Run 219.

Fig. 8 Comparison between the theoretical and the experimental spectra of radiation from shock layer. 


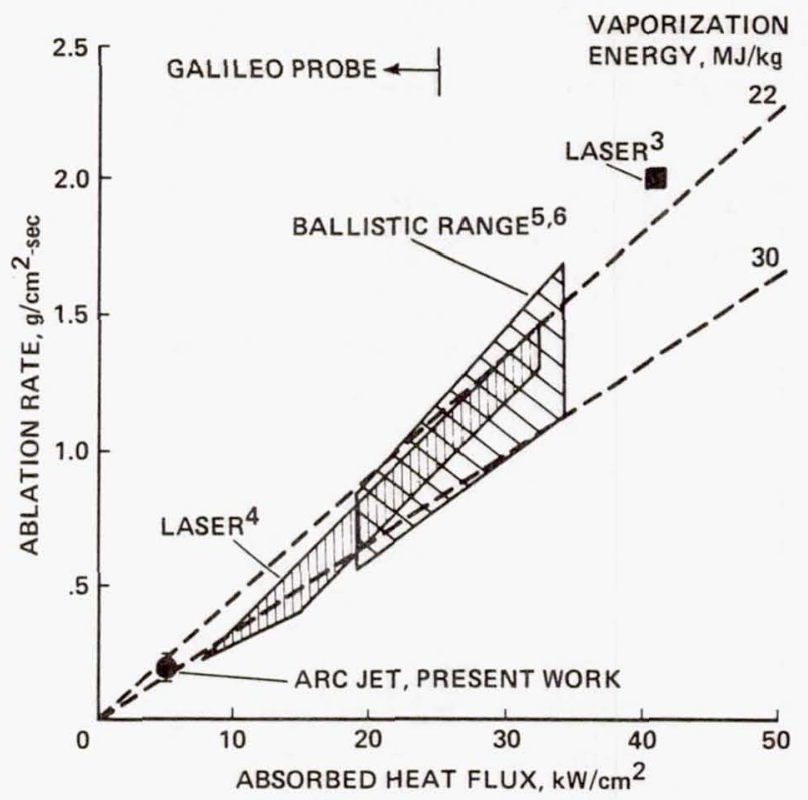

Fig. 9 Comparison among the ablation rates for carbon-phenolic measured by arc-jet, laserirradiation, and ballistic-range methods. 


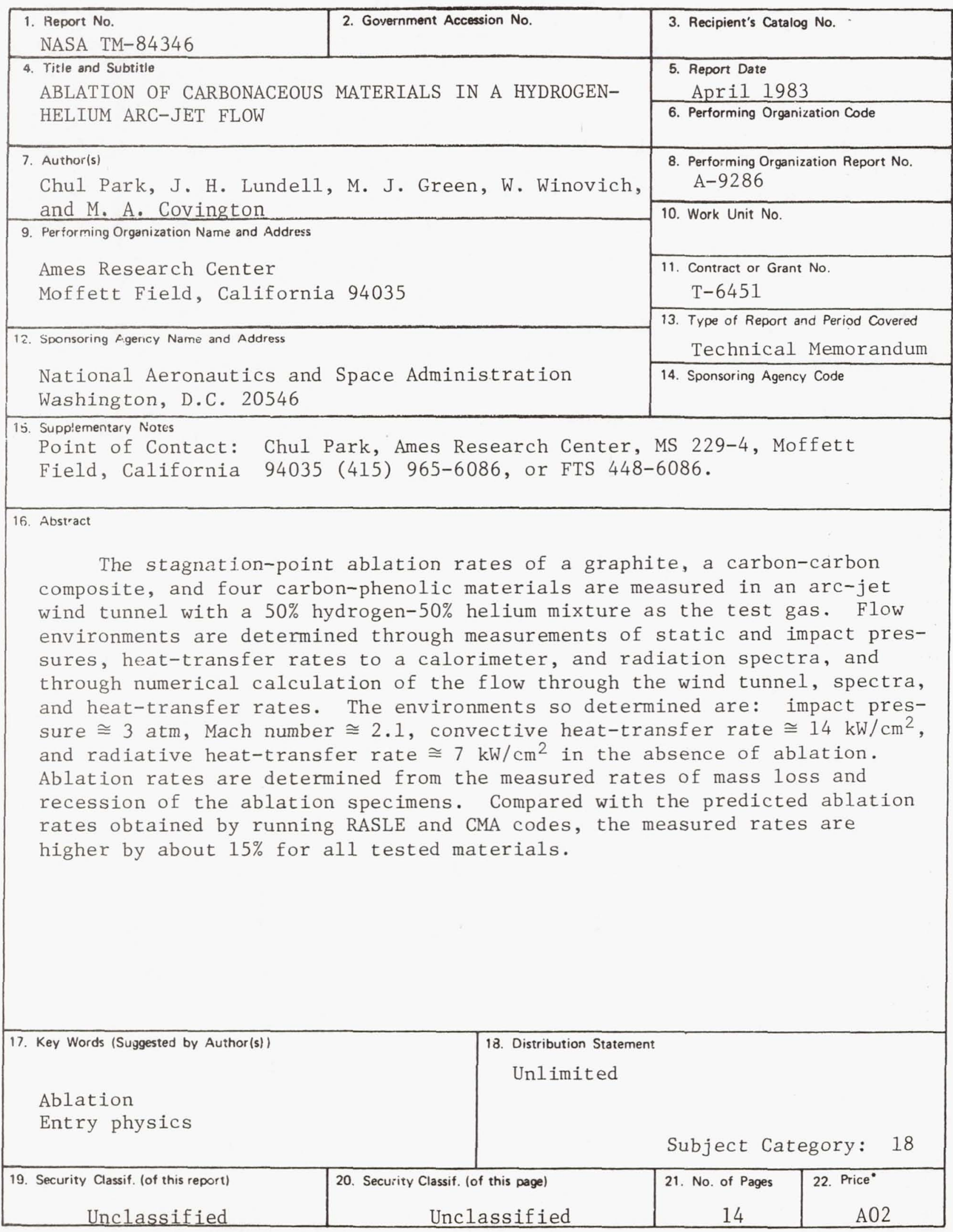

- For sale by the National Technical Information Service, Springfield, Virginia 22161 